\title{
Semantic trajectories data models
}

\author{
Maria Luisa Damiani \\ University of Milan, Italy \\ maria.damiani@unimi.it
}

March 4, 2020

\begin{abstract}
Semantic trajectories is a major paradigm for the representation of movement data, complementary to spatial trajectories. In this article, we introduce key concepts, focusing in particular on the structural properties of semantic trajectories. Hence, we discuss a possible taxonomy of semantic trajectory models, based on their purpose.
\end{abstract}

\section{Introduction}

Semantic trajectories is a major paradigm for representation of the individual movement. Different from spatial trajectories, which are primarily intended to represent the continuous movement of an entity in a coordinated space, e.g. the path of a vehicle, semantic trajectories are grounded on the idea of movement as context evolution, where the context can be any set of features characterizing the situation of the entity in time [6].

Semantic trajectories can represent many different kinds of movement data, such as, series of geo-tagged tweets posted by an individual, series of activities performed in a time frame, paths of vehicles augmented with sensor data on e.g., weather conditions. Abstractly, a semantic trajectory can be seen as a finite sequence of states, where a state is a snapshot of the context in which the movement takes place, typically specifying the individual location together with non-spatial attributes whose values changes in time. The transitions from one state to the next describe the object movement. Actually, the notion of state is instrumental in abstracting a common ground from the variety of existing semantic trajectories representations. A state can be seen as consisting of two components, a time interval $I$, and a description $A$ of the context during $I$. With little abuse of terminology, we refer to $A$ as annotation. Structurally, a semantic trajectory, is a series of states or units, i.e. $\left(I_{1}, A_{1}\right) \ldots\left(I_{n}, A_{n}\right)$.

The paradigm of semantic trajectories encompasses different models, which differ for level of abstraction and purpose. This article attempts to provide a few insights into major research themes and controversial aspects, without any ambition for completeness. The remainder of the article is structured as follows. In order to put the discussion into a proper 
perspective, Section 2 overviews the origins and evolution of the paradigm, reporting as well a few considerations on the relationship between spatial and semantic trajectories. Section 3 elaborates on a simple meta-model of semantic trajectory, to illustrate core concepts. Section 4 presents a possible classification of semantic trajectories models based on their purpose. The article ends up with a discussion on research directions.

\section{Preliminaries}

\subsection{Historical perspective}

For a better understanding of the current research context, we start from some historical notes. Spatial trajectories is the first prominent representation of movement data. Rooted in GPS technology, this representation is at the heart of the Moving Object database model developed in early $2000[9]$.

In the second half of the first decade, novel types of sequential data, other than GPS trajectories, became available, including telecommunication data (e.g., Call Detail Record - CDR data), and series of activities, possibly resulting from a knowledge discovery process. Following this trend, the GeoPKDD project [7], a research project funded by the European Union and carried out by a number of research groups spread across Europe, coined the term semantic trajectory, first appeared in 2007 [1]. The core idea was to enrich spatial trajectories with application-dependent information, possibly extracted from raw mobility data and supplementary data sources through the use of knowledge discovery methods. In the same period, similar concerns were raised in other projects carried out in Asia and US $[26,14]$. Overall, the research in this period is mostly focused on analytical methods and methodologies related to the conceptual modeling and construction of semantic trajectories, e.g [22]. For a survey of early research, we refer the reader to [17].

A more recent stream of research focuses on the data engineering aspects, in particular the management of enriched trajectories through a database system. This line of research is motivated by the growing availability of big enriched trajectory data, heterogeneous and voluminous, calling for efficient processing methods. Especially the plethora of geosocial applications, developed in the last decade, has led to the collection of large amounts of data, e.g. check-in data, naturally organized as sequences of geo-referenced POIs, where a POI (Point of Interest) is a name, possibly accompanied by supplementary information, such as the facility type. Concerning data modeling, we witness the specification of rigorous trajectory models and the systematic development of operational solutions targeting the practical utilization of semantic trajectories. Two contrasting views, however, emerge, the one is application-driven and interprets semantic trajectories primarily as series of time-stamped POIs; the other is application-independent and targets development of generic trajectory models.

The view presented in this article is in line with the latter interpretation, that is a semantic trajectory is any representation of the movement 
as evolving context. In this sense, the term 'semantic trajectories' does not indicate a specific model, but rather a paradigm. In conclusion, semantic trajectories respond to the need of encompassing in a representation situational information made available by novel data acquisition technologies and applications.

\subsection{Spatial vs. semantic trajectories}

We elaborate a bit more on the difference between semantic and spatial trajectories:

Spatial trajectories. Spatial trajectories are built on sequences of timestamped points sampling the entity's movement in a geometric space $\mathcal{S}$, typically the Euclidean or the geographical space. Given a temporal domain $\mathcal{T}$, a spatial trajectory is the sequence: $T=\left(x_{1}, y_{1}, t_{1}\right) . .\left(x_{n}, y_{n}, t_{n}\right)$, with $\left(x_{i}, y_{i}\right) \in \mathcal{S}, t \in \mathcal{T}$. The underlying assumption is that the movement is continuous, namely the location changes smoothly in time, while the sampling rate is sufficiently high so as to ensure a good approximation of the actual movement. Spatial trajectories can be stored in a database, typically as values of suitable data types, and accessed through spatiotemporal query languages $[9,27]$. A standard model of spatial trajectories has been recently proposed by OGC (Open GeoSpatial Consortium) [16].

Semantic trajectories. From the data modeling perspective, we point out three major differences between spatial and semantic trajectories:

- Geometry vs. annotation. Spatial trajectories are grounded on the geometric representation of the object's location. In contrast, semantic trajectories rely on the notion of annotation, which encompasses multiple and diverse kinds of information, including spatial data as a special case. In this sense, the notion of semantic trajectory goes beyond the spatial context.

- Whole vs. part. Spatial trajectories describe the movement of an object as a whole. Therefore, attributes can be only attached to the entire trajectory and not to parts of it. For example, the attribute 'weather' can be only specified for the whole travel. By contrast, a semantic trajectory is an aggregation of smaller components, the units, while the relationship between the unit and the whole trajectory is the mereological relationship of parthood. Annotations can thus be attached to units, and that results in a finer-grained representation of the actual movement.

- Continuous vs. non-continuous. A spatial trajectory provides an approximate representation of a continuous movement taking place in a physical space. In contrast, semantic trajectories can describe a broader range of movements, including discrete and stepwise evolving movements. In this sense, semantic trajectories can provide a framework suitable for a broader spectrum of applications. 


\section{$3 \quad$ A semantic trajectory meta-model}

To exemplify the above concepts, we elaborate on the generic trajectory model sketched in the introductory section. The goal is to provide a simple meta-model sufficiently general to accommodate the diverse interpretations and facilitate the understanding of key concepts.

A simple meta-model. A semantic trajectory $T$ is a sequence of units, where the unit i-th specifies the temporal extent $I_{i}=\left[t_{b}^{i}, t_{e}^{i}\right]$ and the annotation $A_{i}$, i.e. $T=\left(I_{1}, A_{1}\right) . .\left(I_{n}, A_{n}\right)$. Trajectories have the following properties:

- A time interval can degenerate in a single instant, i.e. $t_{b}^{i}=t_{e}^{i}$. In that case the unit is an event. A simplified representation for the event is $\left(t_{i}, A_{i}\right)$

- Time periods $I_{1}, . ., I_{n}$ do not overlap, i.e. $\bigcap_{i} I_{i}=\emptyset$.

- There cannot exist two adjacent periods reporting the same annotation, i.e. every unit contains the maximal interval with identical annotation.

- There can exist temporal discontinuities (gaps), namely consecutive units are not necessarily adjacent in time.

- The annotations are application-dependent. Moreover, an annotation may include spatial data as a special case.

In the following, we show a few examples of annotations and how these can be expressed in terms of the generic model.

Annotations as simple values. Annotations can indicate generic activities, such as tasks (e.g. working, shopping) and transportation means (e.g. train, taxi). Activities have normally a duration, moreover can be represented as attributes of simple type, e.g. string. The movement described by the trajectory has thus the meaning of stepwise evolution in a discrete space. A possible structure for the trajectory unit is: $u_{i}=\left(I_{i},\left[l_{1}, . ., l_{n}\right]\right)$ where $I_{i}$ is the time interval and $\left[l_{1}, . ., l_{n}\right]$ the list of one or more values that, globally, form the annotation. A graphical example of trajectory annotated with strings, specifically reporting the transportation means used by an individual during a trip, is shown in Figure 1.

Annotations as labeled points. A labeled point is a named location. Semantic trajectories annotated with labeled points can describe, for example, the series of check-ins posted by the members of a geo-social network. In that case, the unit model is an event (in the sense given earlier), while the annotation can include the name of the POI, its category (and additional attributes), and the location coordinates. To generalize, a possible structure of the trajectory units is: $u_{i}=\left(t_{i},\left[a t t_{i}^{1}, a t t_{i}^{n},(x, y)_{i}\right]\right)$ indicating: a time-stamp, a sequence of attributes, and a coordinated point, respectively. The graphical representation of semantic trajectory as sequence of labeled points is shown in Figure 2. 


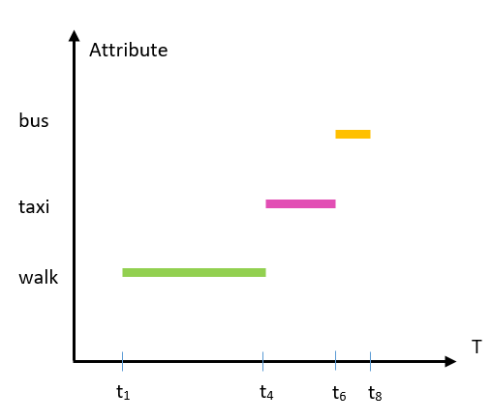

Figure 1: Semantic trajectory as sequence of time-stamped strings

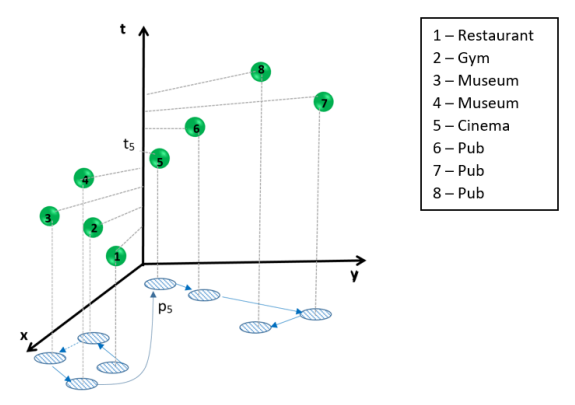

Figure 2: A semantic trajectory as sequence of labeled points

Annotations as spatial sub-trajectories with attributes. In this case, spatial trajectories are split into parts, i.e. segments, based on some partitioning criteria, and each segment is assigned one or more attributes. Attributes can indicate for example the activity performed in the period and the weather conditions. A possible structure for the unit is:

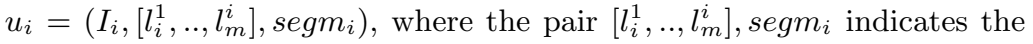
series of attributes, and the segment, respectively. A graphical example of trajectory is reported in Figure 3.

\section{Semantic trajectory data models: a purpose- driven taxonomy}

The paradigm of semantic trajectories encloses a variety of trajectory models. In this section, we discuss a possible classification of the existing models that, either explicitly or implicitly, follow the semantic trajectory paradigm. Models are primarily characterized by the structural properties of trajectories. Such a structure is strongly related to the purpose of the representation. In particular, we identify four main purposes and thus classes, we refer to as: 


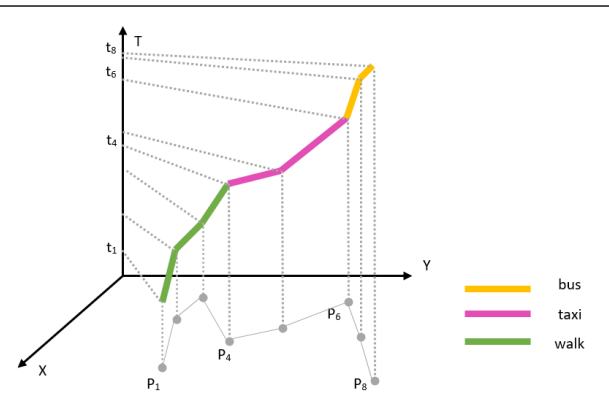

Figure 3: A semantic trajectory as sequence of labeled spatial sub-trajectories

- Conceptual representation

- Database logical model

- Query processing

- Data analytics

In the following, we describe those classes in more detail and show a few representative models.

\subsection{Conceptual representation}

The semantic trajectory models of this class target generic and expressive representations and are exclusively defined at conceptual level. That is, models are built on user-oriented abstractions drawn from application practices and requirements, while the operational aspects, for example which operations can be performed over semantic trajectories, are not taken into account. This class comprises two main groups of models, those based on stop-and-move patterns and those based on episodes, respectively, described as follows.

Models based on stops-and-moves. The seminal work in [18] was inspired by the observation that moving individuals often alternate periods of relative stationarity (stops) with periods of mobility (move). Goal of the model was to capture that mobility behavior by annotating spatial trajectories with labels denoting stops and moves.

Stop-and-move is a pattern of practical relevance in a variety of phenomena. For example, in an urban setting, the stops can represent POIs, while the moves the transportation means used by individuals for moving from one stop to another; in the field of animal ecology, the stops can indicate the animal home-ranges, while the moves the seasonal migrations. To convey the intuition, an example of trajectory consisting of stops and moves is illustrated in Figure 4. Following the traditional approaches to conceptual modeling, this model is expressed using a graphical notation. The substantial limitation of this model (and alike) is that it is built on a specific mobility pattern. 


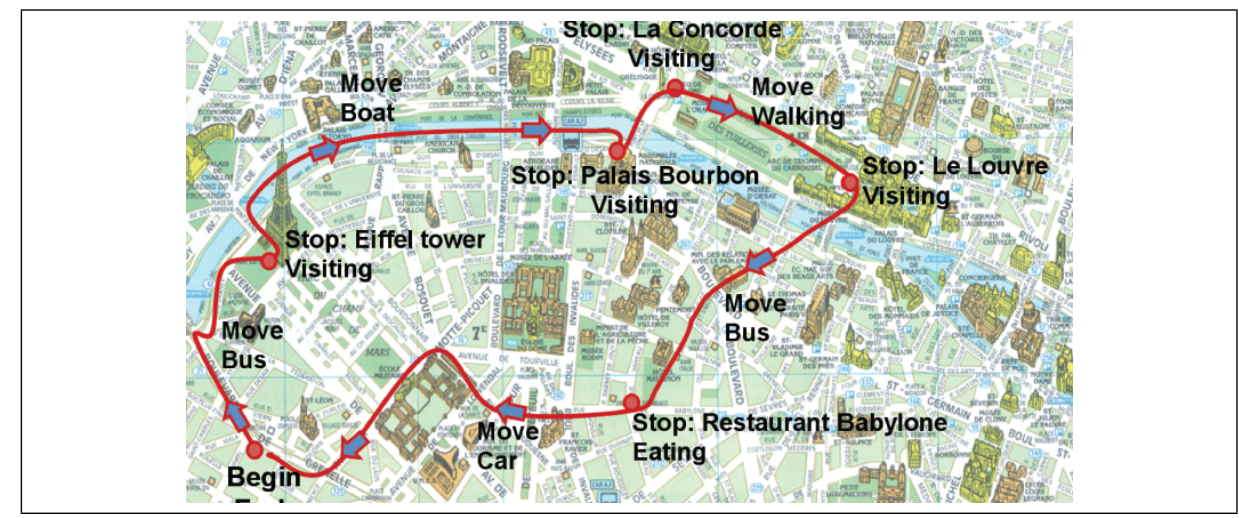

Figure 4: A semantic trajectory consisting of stop and moves [17]

Models based on episodes. The models of this group aim to overcome the above limitations, by introducing generic concepts not confined to any specific mobility pattern. The core idea is to enrich spatial trajectories with supplementary information, i.e. numerical and categorical attributes, which can regard either the single points of the trajectories, or the segments resulting from a prior operation of trajectory partitioning. In the latter case, the segments are called episodes and are associated with a time interval [17]. In this view, stops and moves can be seen as specific types of episodes. The operation of segmentation turns the continuous movement, represented by the spatial trajectory, into a stepwise movement. A semantic trajectory can specify one or multiple segmentations. For example, the trajectory representing a travel can be partitioned based on the transportation means used by the individual and weather conditions. This generalization provides the basis for extended conceptual models, e.g. $[2,15]$.

\subsection{Database logical models}

Another class of semantic trajectory models are those utilized in databases. In this case, the goal is not only to devise a rich representation, but also to provide appropriate techniques and languages for the manipulation and querying of trajectories through a database system. In general, the use of rich-content data models is paid in terms of complexity, usability, efficiency. Simply, the more expressive the representation, the more complex the operational system. Therefore, a key challenge is to find a trade-off between expressivity and cost of the solution. Another aspect of concern is how to embed the trajectory data model into an existing and extensible database system, so as to make the system usable in real applications. Two major models of this class are symbolic trajectories, and its evolution, multi-attribute trajectories, discussed next.

Symbolic trajectories. Symbolic trajectories $[19,4,10]$ describe a movement that evolves step-wise, not necessarily in a physical space. 
In its basic form, a symbolic trajectory consists of a series of units: $\left(I_{1}, l_{1}\right), \ldots,\left(i_{n}, l_{n}\right)$ where $I_{j}$ is a time interval and $l_{j}$ a single label (i.e., a name) or, in alternative, a set of labels. Units can be also annotated with one or more places, where a place is a labeled point. An example of symbolic trajectory, reporting a series of activities [5], is as follows:

$$
\text { ([8:45 - 17:00) working) }([17: 00-18: 30] \text { shopping) (..) }
$$

Key feature of the model is the pattern-based query language, for the retrieval of trajectories based on pattern matching and rewriting. Matching is used to retrieve the trajectories satisfying a pattern, while rewriting is to extract or redefine parts of trajectories matching the given pattern. A pattern is sequential, namely it consists of a series of simple patterns that are to be matched in the given order. Moreover, patterns are defined using regular expressions, variables, and predicates. In particular, variables are used to verify predicates beyond the scope of a simple pattern [5]. For example a query containing a variable $(\mathrm{X})$ and a condition on the variable (the condition following //) is the following:

$\mathrm{Q}$ : * (morning working) $\mathrm{X}$ (_ shopping)*//duration X.time $>2 *$ hour

In this example, the trajectories matching $Q$ are those in which the working activity takes place in the morning and is followed by a shopping activity taking more than 2 hours. For the language syntax, we refer the reader to [10].

Another feature is that the pattern language and the type system are integrated into an extensible moving object database [10]. In particular, a symbolic trajectory is a value of type mlabel (or mlabels for sets of labels), or mplace (and mplaces), wherein the construct $m$ type is a shortcut of moving(type), defining a mapping from the time domain to the domain of the given type. Hence, a value of type mlabel indicates a time-varying string. As an example, we can construct a relation describing the trips of individuals through an attribute of type mlabel specifying the road along which the user is traveling:

CREATE TABLE Trips (Id: int, Trip: mlabel)

This model suffers from a major limitation, that the annotation is exclusively textual.

Trajectories with multiple attributes. This data model [20] responds to the limitations of symbolic trajectories, by extending such a model with annotations consisting of multiple attributes of arbitrary timevarying type. Time-varying types include mpoint (i.e. a time-varying point representing a spatial trajectory) and mreal/mint (i.e., a time varying numeric quantity). In essence, the idea is to annotate a time interval with a tuple of attribute values, where the attributes can be of different type, either spatial or not. As an example, the Trips table can have, as attributes, a spatial trajectory describing the actual path, the time-varying road name, and the time-varying speed limit along the road, as shown below:

CREATE TABLE Trips (Id: int, Path: mpoint, RoadName: mlabel, SpeedLimit: mint) 
In this example, the non-spatial attributes, i.e. road name and speed limit, are categorical attributes that evolve step-wise, inducing two different segmentations of the spatial trajectory. Sequential queries over multi-attribute trajectories are formulated through a slightly extended pattern-based language, in which variables are associated to tuple-based units.

\subsection{Query processing}

The models in this class are instrumental to the efficient computation of selected types of queries over large datasets of trajectories. This class also includes intermediate representations, possible resulting from a preprocessing step. In this sense, these models are possibly defined ad-hoc. In the following, we outline two representative approaches dealing with different types of semantic trajectories and focusing on specific types of query.

Top-K spatial keyword queries over trajectories of labeled locations. The overall goal is the efficient retrieval of semantic trajectories defined as activity trajectories [24]. An activity trajectory is a series of timestamped POIs, supplemented with textual keywords describing the activities undertaken at that place. Structurally, a trajectory consists of units representing events (in the sense of Section 3) annotated with a POI $p$ and with a set of keywords $h_{1}, . ., h_{m}$, i.e., $u=\left(t, p,\left\{h_{1}, . . h_{m}\right\}\right)$. Given a query $\mathrm{q}=(x, y, t w, q w, a)$ specifying a location $(x, y)$, a time interval $t w$, a set of keywords $q w$ and a weight factor $a$ used as preference, the query retrieves the $k$ trajectories that are most similar to the query, based on a criteria of similarity encompassing spatial distance, keywords matching and places popularity. Example: the user located in $p$ is willing to eat Japanese food, watch movie and go to a pub. The user does not know which places are more convenient to visit, thus search for the trajectories reporting similar travel experience by users moving nearby. Query processing is supported by an augmented R-tree index, called ITB-tree, supporting both spatial and keyword search.

Sequential range queries over labeled spatial sub-trajectories. In this case, the goal is to efficiently retrieve spatial trajectories augmented with textual annotations. Specifically, a semantic trajectory is a sequence of labeled segments, possibly resulting from a prior temporal overlay of a spatial trajectory with a symbolic trajectory [10]. The units of the trajectory are triples $\left(I_{i}, l_{i}\right.$, seg $\left._{i}\right)$, where the pair $l_{i}$, seg $_{i}$ consists of a spatial trajectory segment and a label, respectively [11].

A sequential range query $q$ is a sequence of simple queries, $q=q_{1}, . ., q_{n}$, each specifying a range constraint on time, space and labels, which are to be solved in the given order. Example: find the users traveling by car or by bus and passing by region $\mathrm{A}$ in the morning and by region $\mathrm{B}$ in the evening. In this query, $\mathrm{A} / \mathrm{B}$ define the spatial range, morning/evening the temporal range and car/bus the label range. The query semantics is defined as 
follows: a trajectory satisfies the query $q$ if there exist $n$ instants $t_{1}, . ., t_{n}$ with $t_{i}<t_{i+1}$ such that for every instant $t_{i}$, there exists a trajectory unit matching $q_{i}$ [11]. Importantly, the semantics, and thus the result of the query, does not depend on the distribution of the sample points in the spatial trajectory. Query processing is supported by an hybrid index, called IRWI (IR index with Trajectory Identifiers), an augmented 3D RTree integrating space, time and label search. Moreover the input query is processed by evaluating concurrently every simple query of the sequence during the traversal of the IRWI tree.

\subsection{Data analytics}

The last class of trajectory models are those employed for data analytics purposes. These models are application dependent, namely the structural properties of the model do not result from an abstraction effort, but rather are drawn from the application problem. These trajectories are, therefore, grounded on real application needs, and, for that reason, interesting for the discussion. Since this is a broad area, we limit ourselves to consider two recent examples of analytical problems on semantic trajectories, while we refer the reader to [25] for a survey:

Frequent sequential pattern mining. The general goal is to respond to queries such as: Where do people usually go to relax after work? [23, 3]. A specific instance of the problem considers trajectories structured as sequence of events annotated with categorized POIs (e.g., restaurant) [23]. In more detail, the mining task is to extract from trajectories, sequences of groups of POIs satisfying a number of constraints, in particular, the members of the groups shall be semantically homogeneous and spatially close; and the groups shall be visited in a specific order and satisfying a maximum transition time. The sequences matching those conditions are the sequential patterns. . The ultimate goal is to find those sequential patterns that are frequent in the trajectory dataset.

Topical trajectory pattern mining [12]. The problem is related to the analysis of large collections of geo-tagged messages posted through micro-blogging services such as Twitter. The input dataset consists of geo-tagged message trajectories, namely trajectories reporting the series of time-stamped and geo-referenced messages posted by users. In more detail, a trajectory consists of units representing events, where the annotation consists of a coordinated point $(\mathrm{x}, \mathrm{y})$, i.e. the user position, and a set of words taken from a given vocabulary, and extracted from the actual text message. The mining task is to identify the geographic locations, where geo-tagged messages are posted with the same topic (semantic regions), and the transitions between semantic regions.

\section{$5 \quad$ Final remarks and research directions}

In summary, the paradigm of semantic trajectories, encompasses a large variety of models defined at different levels of abstraction and for differ- 
ent purpose. Semantic trajectories are, thus, characterized by a great structural heterogeneity. Overcoming such fragmentation is a challenge. Another major goal is to provide workable solutions allowing for an effective utilization of large datasets of semantic trajectories. Although a few systems are available, e.g. $[20,8,15]$, the engineering efforts are still limited.

Concerning the structural properties of trajectories, the 'semantics' of the movement is often expressed by textual or spatial keywords embedded as annotations. While the integration of text, space and time raises interesting research issues, dealing with keywords only might be not sufficient to fulfill the requirements posed by modern applications, e.g. IoT. In essence, what is needed is the capability of describing the context evolution through multiple dimensions. The recent database model of multi-attribute trajectory [20] is in line with that view, similarly the notion of multi-aspect trajectory in [15]. Several issues, however, remain to be addressed, including: the specification of a unifying framework enabling the coherent representation of both discrete, stepwise and continuous movement; the specification of query processing mechanisms over multi-dimensional trajectories, e.g. [21]; the efficient processing of complex operations, such as semantic trajectories join. All these functionalities are instrumental in supporting trajectory data analytics and, more in general, the research and technological stream of mobility data science. From a data representation perspective, a challenging direction is towards representation learning methods to support complex tasks such as trajectory similarity detection [13], applied to semantic trajectories. In this perspective, the availability of a solid and flexible semantic trajectory representation framework paves the way to novel research opportunities on fine-grained behavior analysis and prediction.

Acknowledgments. This work is partially supported by the Italian government via the NG-UWB project (MIUR PRIN 2017).

\section{References}

[1] L.O. Alvares, V. Bogorny, B. Kuijpers, J. de Macedo, B. Moelans, and A. Vaisman. A model for enriching trajectories with semantic geographical information. In Proc. ACM GIS, 2007.

[2] V. Bogorny, C. Renso, A. R. de Aquino, F. L. de Siqueira, and L. O. Alvares. CONSTAnT - a conceptual data model for semantic trajectories of moving objects. Trans. GIS, 2013.

[3] D-W Choi, J. Pei, and T. Heinis. Efficient mining of regional movement patterns in semantic trajectories. Proc. VLDB Endow., 10(13):2073-2084, 2017.

[4] M. L. Damiani and R. H. Güting. Semantic trajectories and beyond. In Proc. IEEE MDM, 2014.

[5] M. L. Damiani, H. Issa, R. L. Güting, and F. Valdés. Symbolic trajectories and application challenges. SIGSPATIAL Special, 7(1):51-58, 2015. 
[6] Anind K. Dey. Understanding and using context. Personal and Ubiquitous Computing, 5(1), 2001.

[7] F. Giannotti and D. Pedreschi. Mobility, Data Mining and Privacy: Geographic Knowledge Discovery. Springer Publishing Company, Incorporated, 1 edition, 2008.

[8] F. Gryllakis, N. Pelekis, C. Doulkeridis, S. Sideridis, and Y. Theodoridis. Spatio-temporal-keyword pattern queries over semantic trajectories with hermes@neo4j. In Proc. EDBT, 2018.

[9] R. H. Güting, M. H. Böhlen, M. Erwig, C. S. Jensen, N. A. Lorentzos, M. Schneider, and M. Vazirgiannis. A foundation for representing and querying moving objects. ACM Trans. Database Syst, 2000.

[10] R.H. Güting, F. Valdés, and M. L. Damiani. Symbolic trajectories. ACM Transactions on Spatial Algorithms and Systems, 1(2):7, 2015.

[11] H. Issa and M. L. Damiani. Efficient access to temporally overlaying spatial and textual trajectories. In IEEE MDM, 2016.

[12] Y. Kim, J. Han, and C. Yuan. Toptrac: Topical trajectory pattern mining. In Proceedings of KDD, 2015.

[13] X. Li, K. Zhao, G. Cong, C. Jensen, and W. Wei. Deep representation learning for trajectory similarity computation. In Proc. ICDE, 2018.

[14] J. Liu, O. Wolfson, and H. Yin. Extracting semantic location from outdoor positioning systems. In Proceedings of the 7th International Conference on Mobile Data Management, page 73, 2006.

[15] R. Santos Mello, V. Bogorny, L. O. Alvares, L. Zambom Santana, C. Ferrero, A. Frozza, G. A. Schreiner, and C. Renso. Master: A multiple aspect view on trajectories. Transactions on GIS, 2019.

[16] OGC. Moving features encoding part 1: Xml core. http://docs.opengeospatial.org/is/18-075/18-075.html, 2019.

[17] C. Parent, S. Spaccapietra, C. Renso, G. Andrienko, N. Andrienko, V. Bogorny, M. L. Damiani, A. Gkoulalas-Divanis, J. Macedo, N. Pelekis, Y. Theodoridis, and Z. Yan. Semantic trajectories modeling and analysis. ACM Comput. Surv., 2013.

[18] S. Spaccapietra, C. Parent, M. L. Damiani, J. A. F. de Macêdo, F. Porto, and C. Vangenot. A conceptual view on trajectories. Data Knowl. Eng., 65(1):126-146, 2008.

[19] F. Valdés, M.L. Damiani, and R.H. Güting. Symbolic Trajectories in Secondo: Pattern Matching and Rewriting. In DASFAA, 2013.

[20] F. Valdés and R. H. Güting. A framework for efficient multi-attribute movement data analysis. VLDB J., 28(4):427-449, 2019.

[21] J. Xu, Z. Bao, and H. Lu. Continuous range queries over multiattribute trajectories,. In IEEE International Conference on Data Engineering (ICDE), 2019.

[22] Z. Yan, D. Chakraborty, C. Parent, S. Spaccapietra, and K. Aberer. Semantic trajectories: Mobility data computation and annotation. ACM Trans. Intell. Syst. Technol., 2013. 
[23] C. Zhang, J. Han, L. Shou, J. Lu, and T. La Porta. Splitter: Mining fine-grained sequential patterns in semantic trajectories. Proc. VLDB Endow., 7(9):769-780, May 2014.

[24] K. Zheng, B. Zheng, J. Xu, G. Liu, A. Liu, and Z. Li. Popularityaware spatial keyword search on activity trajectories. World Wide Web, 20(4):749-773, 2016.

[25] Y. Zheng. Trajectory data mining: An overview. ACM Trans. Intell. Syst. Technol., 6(3), May 2015.

[26] Y. Zheng, L. Wang, R. Zhang, X. Xie, and W. Ma. Geolife: Managing and understanding your past life over maps. In Proc. IEEE MDM, 2008.

[27] Y. Zheng and X. Zhou. Computing with Spatial Trajectories. Springer Publishing Company, Incorporated, 2011. 